Article

\title{
Selective Growth of $\alpha$-Sexithiophene by Using Silicon Oxides Patterns
}

\section{Cristiano Albonetti ${ }^{1, *}$, Marianna Barbalinardo ${ }^{1}$, Silvia Milita ${ }^{2}$, Massimiliano Cavallini ${ }^{1}$, Fabiola Liscio $^{2}$, Jean-François Moulin ${ }^{3}$ and Fabio Biscarini ${ }^{1}$}

1 CNR-ISMN, Institute for the Study of Nanostructured Materials, Via P. Gobetti 101, Bologna I-40129, Italy; E-Mails: m.barbalinardo@bo.ismn.cnr.it (M.B.); m.cavallini@bo.ismn.cnr.it (M.C.); f.biscarini@bo.ismn.cnr.it (F.B.)

2 CNR-IMM, Institute for Microelectronics and Microsystems, Via P. Gobetti 101, Bologna I-40129, Italy; E-Mails: milita@bo.imm.cnr.it (S.M.); liscio@bo.imm.cnr.it (F.L.)

3 GKSS, Forschungszentrum Geesthacht GmbH, Geesthacht D-21502, Germany; E-Mail: jean-francois.moulin@hzg.de

* Author to whom correspondence should be addressed; E-Mail: c.albonetti@bo.ismn.cnr.it; Tel.: +39-0516398531; Fax: +39-0516398540.

Received: 1 August 2011; in revised form: 25 August 2011 / Accepted: 29 August 2011 / Published: 6 September 2011

\begin{abstract}
A process for fabricating ordered organic films on large area is presented. The process allows growing sexithiophene ultra-thin films at precise locations on patterned $\mathrm{Si} / \mathrm{SiO}_{x}$ substrates by driving the orientation of growth. This process combines the parallel local anodic oxidation of $\mathrm{Si} / \mathrm{SiO}_{x}$ substrates with the selective arrangement of molecular ultra-thin film. The former is used to fabricate silicon oxide arrays of parallel lines of $400 \mathrm{~nm}$ in width over an area of $1 \mathrm{~cm}^{2}$. Selective growth arises from the interplay between kinetic growth parameters and preferential interactions with the patterned surface. The result is an ultra-thin film of organic molecules that is conformal to the features of the fabricated motives.
\end{abstract}

Keywords: sexithiophene; atomic force microscopy; pattern; template; annealing 


\section{Introduction}

New strategies for fabricating planar organic devices by placing molecules in a controlled way are the key requirements to realize electronic, optical, and magnetic devices and sensors [1]. Single molecules [2] and atoms [3] were moved, positioned and used to fabricate prototype devices for investigating the physics at the atomistic length scale. On the other hand, the electrical performances of standard organic devices [4] with micrometric size are mainly influenced by cooperative phenomena amongst molecules that occur at the nanometric/micrometric length scale. Accordingly, strategies for organizing molecules at these length scales are necessary. Unconventional lithographic techniques [5] have successfully re-organized molecular films into micrometric/nanometric structures for tuning optical [6] and electrical [7] properties, but one of the major aims of nanotechnology is to assemble devices by driving the self-organization process of molecules. The advantage of the latter approach is two-fold. On one hand, the possibility to implement additive manufacturing [8], viz. the usage of the optimum amount of material needed for the performance of the device; on the other hand, the capability of tailoring the relevant properties of those devices, such as charge mobility [9], spin coherence length [10], or radiative relaxation [11] by controlling the lateral size of the active material from nanometer to micrometer length scale. Since the lengths scales of physical phenomena in conjugated materials are in the nanometer range, optimizing the organization of the molecules at the nanometer scale would lead to an enhancement of the device's physical properties.

Currently, few examples of fabrication approaches for conjugated materials are reported in literature [12-14]. Organic Molecular Beam Deposition (OMBD) is the most consolidated thin film growth technique for organic electronics, but it is lacking in the control of lateral size of the domains due to the coalescence of uncorrelated domains [15].

In this article we report a process for growing selectively $\alpha$-sexithiophene (6T) molecules on silicon oxide patterns in order to fabricate molecularly ordered nanostructures on large superficial area $\left(\mathrm{cm}^{2}\right)$. The method yields monolayer stripes, whose width is a few hundred nanometers. These low-dimensional structures are grown at predetermined positions on the substrate. Their shape and size are controlled by $\mathrm{SiO}_{x}$ patterns fabricated on the substrate. The molecules on the pattern maintain their orientation with their long axis normal to the substrate, viz. the same orientation as those forming islands on non-patterned surface. Morphological, structural and thermal properties of $6 \mathrm{~T}$ nanostructures were investigated by means Atomic Force Microscopy (AFM) and X-ray scattering.

\section{Experimental and Methods}

\subsection{Fabrication of $\mathrm{SiO}_{x}$ Patterned Surfaces}

Nanometric patterns on $\mathrm{Si}_{/} / \mathrm{SiO}_{x}$ (native) substrates were fabricated by using the parallel-local anodic oxidation (PLAO) technique [16]. This method yields silicon oxide nanostructures with height variable from few $\mathrm{nm}$ to $15 \mathrm{~nm}$ [17]. The size of the stamp $\left(1 \mathrm{~cm}^{2}\right)$ enables us to pattern the surface across centimeter length scale. To implement this technique, we built a machine to bring a metallized polydimethylsiloxane (PDMS) stamp in contact with the silicon surface. The PDMS stamps (Sylgard 184 Down Corning) were prepared by replica molding [18] using, as a master, the surface of a blank compact disk (CD). On the centimeter length scale, the $\mathrm{CD}$ is composed of an array of parallel stripes 
with spatial periodicity $(\lambda)$ of $1.4 \mu \mathrm{m}$, full width at half maximum (FWHM) of $400 \mathrm{~nm}$ and height $(h)$ of $200 \mathrm{~nm}$.

The machine, with prearranged stamp and substrate, was inserted in a sealed chamber with controlled relative humidity. The stamp and the sample were gently placed in contact by means of the downward motion of a micrometric screw. The oxide nanostructures (stripes-Figure 1a) were fabricated when a bias voltage of $36 \mathrm{~V}$ is applied between the stamp and the silicon substrate for $2 \mathrm{~min}$, with a relative humidity of $90 \%$. The flexibility of the stamp enables a homogeneous conformal contact with the silicon surface, resulting in an excellent reproducibility of the process. In addition, this allowed us to fabricate a complex nanostructure (grid-Figure 1b) by means of two subsequent oxidations with the same stamp (just rotating the sample of $90^{\circ}$ ).

Figure 1. $\mathrm{Si}$ oxide patterns on $\mathrm{SiO}_{x}$ surfaces fabricated by using parallel-local anodic oxidation (PLAO) technique. (a) Array of Si oxide stripes with $\lambda=1.28 \mu \mathrm{m}$, full width at half maximum $(\mathrm{FWHM}) \approx 400 \mathrm{~nm}$ and $h=(74 \pm 4) \AA$; and (b) Si oxide grid (holes size $\approx 650 \mathrm{~nm}$, bar width $\approx 500 \mathrm{~nm}$ ) fabricated by turning of $90^{\circ}$ the $\mathrm{Si} / \mathrm{SiO}_{x}$ substrate.

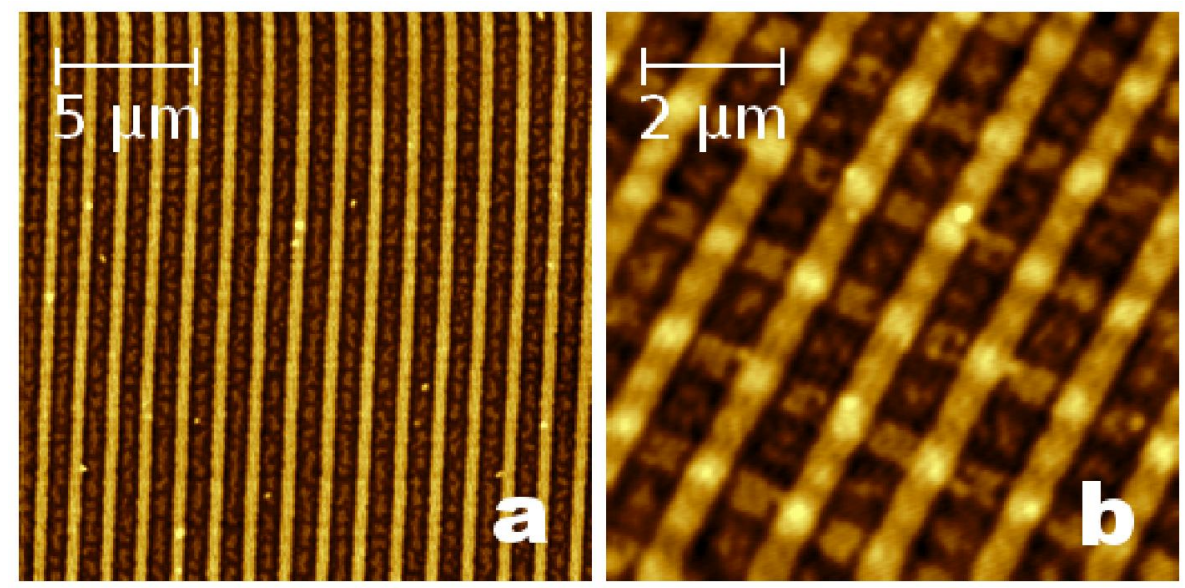

\subsection{Organic Molecular Beam Deposition of 6T Ultra-Thin Films}

Ultra-thin films of 6T molecules (Sigma-Aldrich) were grown by OMBD in high vacuum (base pressure $2 \times 10^{-8}$ mbar) [19]. The film thickness $t$ ranges between $10 \AA$ (surface coverage $\Theta \approx 40 \%$, i.e., sub-monolayer thick film) and $200 \AA(\approx 8$ monolayer (ML)) [20]. Substrates used were Si $\left(n^{++}\right.$-type, doping $\left.>5 \cdot \times 10^{19} \mathrm{~cm}^{-3}, \rho \leq 0.0015 \Omega \cdot \mathrm{cm}\right)$ with native $\mathrm{SiO}_{x}$ either patterned $(\mathrm{P})$ or non-patterned (NP), held at $120{ }^{\circ} \mathrm{C}$ during the molecular deposition [21]. The deposition rate $r$ was kept constant at a value $\approx 0.04 \AA / \mathrm{s}$. Both $t$ and $r$ were measured in-situ with a quartz crystal micro balance (QCM) and cross-checked with Atomic Force Microscopy (AFM) measurements.

A custom OMBD system was home-built to perform in-situ and in real-time X-ray measurements on growing 6T films (Figure 2a). 6T molecules were sublimed under high vacuum (base pressure $2 \times 10^{-6}$ mbar) by using a specially designed Knudsen cell (Figure $2 \mathrm{~b}$ ). 
Figure 2. (a) Upper part of the Organic Molecular Beam Deposition (OMBD) system with custom-tailored flange (CF100) consisting of sample heater (3), shutter (2) and quartz crystal micro balance (QCM) (1) with water cooling circuit (on the right). The flange is mounted on a custom-tailored reduction from CF100 to CF40 (4) with three kapton windows because kapton is X-ray transparent; and (b) Special designed Knudsen cell (5) with motorized shutter (6). The complete OMBD system is compactly furnished, just $50 \mathrm{~cm}$ high and $25 \mathrm{~cm}$ wide.

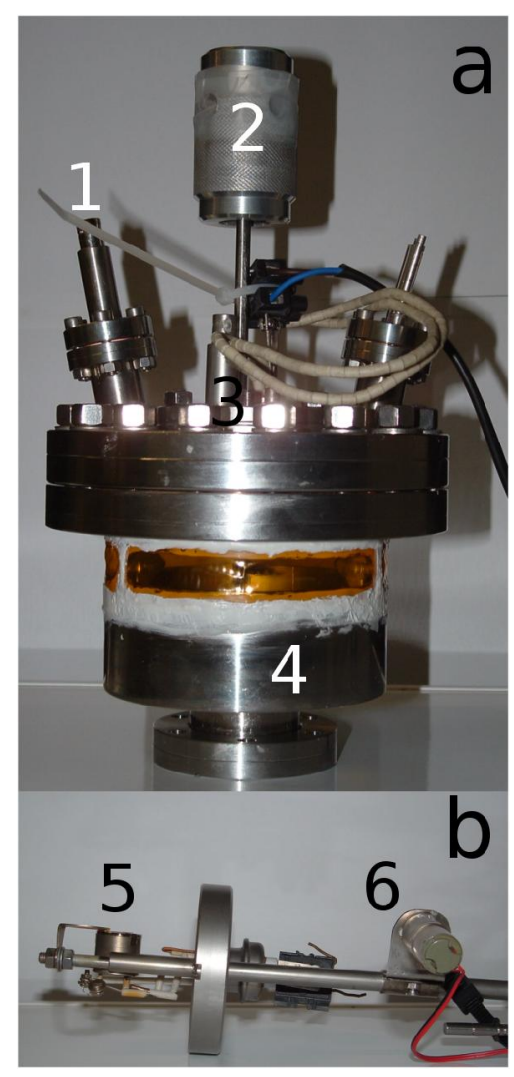

\subsection{Atomic Force Microscopy Measurements}

The AFM employed is a Smena model (NT-MDT, Moscow, Russia) working in Intermittent Contact Mode (ICM) [22]. Cantilevers for ICM were silicon cantilevers (NSG11-NT-MDT, two cantilevers for chip) with elastic constant $k=12(6) \mathrm{N} / \mathrm{m}$ and a resonance frequency $\omega_{0}=255(150) \mathrm{kHz}$.

The topography of 6T films was investigated by AFM under ambient conditions, as well as their thermal stability [23]. The sample was directly attached to the top of a heating stage (maximum temperature $T_{\max }=200{ }^{\circ} \mathrm{C}$ ) via silver paint (SPI supplies). The substrate temperature $T_{\text {sub }}$ was raised stepwise from RT to $120{ }^{\circ} \mathrm{C}\left(\Delta T\right.$ steps of $10{ }^{\circ} \mathrm{C}$ ) and held one hour in order to reach the thermal equilibrium of the cantilever-sample system. $6 \mathrm{~T}$ film was topographically characterized in ICM for increasing $T_{\text {sub }}\left(\mathrm{RT}, 30{ }^{\circ} \mathrm{C}, 40^{\circ} \mathrm{C} \ldots\right)$. 


\subsection{Image Processing of 6T Islands: Angular Orientation, Roughness, Relative Position and} Surface Coverage

$6 \mathrm{~T}$ molecules deposited on chemically inert, $\mathrm{NP}, \mathrm{SiO}_{x}$ surface, firstly form stable nucleus of molecules that evolve as islands during the deposition (Figure 3a) and lastly coalesce one each other to form a continuous organic film [24,25]. Conversely, molecules deposited on $\mathrm{P}_{\mathrm{SiO}_{x}}$ surface form elongated islands in the stripes direction where molecules are mainly grew on top of the $\mathrm{SiO}_{x}$ stripes (Figure 3b).

6T islands grown on both $\mathrm{P}$ and $\mathrm{NP} \mathrm{SiO}_{x}$ surfaces were imaged by $\mathrm{AFM}$ and morphologically analyzed by measuring: (a) angular orientations with respect to a preferential direction (morphological effect induced by stripes); (b) roughness and relative position induced by the $T_{\text {sub }}$ changes; and (c) $\Theta$ evolution. AFM images were quantitatively analyzed by using Gwyddion image software (version 2.25) [26].

Figure 3. $6 \mathrm{~T}$ sub-monolayer film $(\approx 0.4 \mathrm{ML})$ grown on $\mathrm{Si} / \mathrm{SiO}_{x}$ surface where the half of the surface was patterned. AFM images of 6T islands on NP (a) and P (b) surfaces. (c) Maximum and minimum bounding dimensions and angles of a grain (courteously from reference [27]).
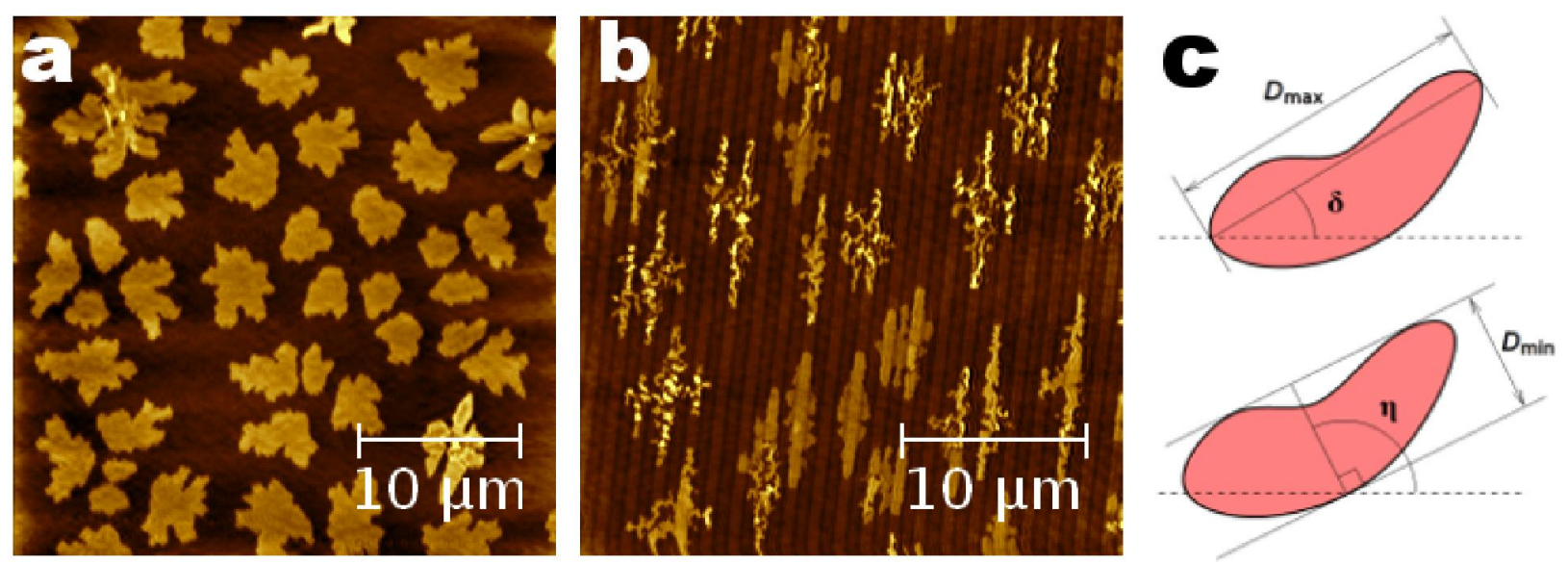

The angular orientations were measured by using the Grain Analysis toolbox of Gwyddion [27]. Firstly, 6T islands were indentified by means of a height threshold algorithm. Regardless of the island's shape, maximum $\left(D_{\max }\right)$ and minimum $\left(D_{\min }\right)$ bounding dimensions were defined and associated to the longest dimension and the maximum width (orthogonal to $D_{\max }$ ) of 6T islands (Figure 3c. Accordingly, two angles $\left(\delta\right.$ and $\eta=90^{\circ}+\delta$ ) were defined with respect to the horizontal direction (dashed lines in Figure 3c).

AFM images were digitally rotated for matching the direction of the stripes to the horizontal one and $\delta$ (in degrees $-^{\circ}$ ) was measured as the angle between this direction and $D_{\max }$. Data were plotted in polar graph where the $x$-axis runs from $-90^{\circ}$ to $90^{\circ}\left(0^{\circ}\right.$ corresponds to a perfect alignment between island and stripe) and the $y$-axis represents the ordinal number of islands. The degree of the island's alignment was quantified through the Order Parameter (S), as calculated from the equation [28]: 


$$
S=\frac{3}{2}<\cos ^{2} \delta>-\frac{1}{2}
$$

where $S$ is the mean value of the second Legendre Polynomial (dimensionless). In particular, $S=1$ corresponds to a perfect alignment of 6T islands along the stripes, while if $S$ goes to 0 islands have an isotropic distribution on the surface.

The Root Mean Square Roughness $R_{\mathrm{q}}$ of both $\mathrm{SiO}_{x}$ surface and top surfaces of islands was measured by Gwyddion. It is defined as the average of the measured height deviations taken within the evaluation length and measured from the mean line.

The relative spatial position of each island was measured by using the software ImageJ (version 1.44p) [29]. The geometric centre of a $6 \mathrm{~T}$ crystal (C) was taken as a reference point due to its geometrical stability on $T_{\text {sub }}$ changes. Straight lines were drawn to connect $\mathrm{C}$ with the geometric centers of the surrounding $6 \mathrm{~T}$ islands and their lengths were measured by ImageJ. Such analysis was performed on AFM images attained at RT, 50, 70, 80, 90, 110 and $120^{\circ} \mathrm{C}$.

The surface coverage $\Theta$ was calculated by using the height threshold algorithm to identify the $6 \mathrm{~T}$ islands. For definition, $\Theta$ is the ratio between the sum of the islands areas projected on the $\mathrm{SiO}_{x}$ surface and the total area of the image.

\subsection{In-Situ and Real-Time X-Ray Measurements}

X-ray scattering measurements in-situ and in real-time were performed during the deposition of 6T molecules on $\mathrm{P}$ (stripes) and $\mathrm{NP} \mathrm{Si} / \mathrm{SiO}_{x}$ substrates $\left(1 \mathrm{~cm}^{2}\right)$. X-ray experiments were performed at the ID32 beam line of the European Synchrotron Radiation Facility (ESRF-Grenoble, France) by using the OMBD system (cf. 2.2) mounted on a horizontal Huber 6-circle diffractometer [30].

A Si(111) double crystal monochromator was used to select the $0.6667 \AA$ wavelength. A set of vertical $(v)$ and horizontal $(h)$ slits were used to define the beam size $(v=400 \mu \mathrm{m}, h=20 \mu \mathrm{m})$. Such small $h$ reduces the X-ray damages of the organic film [31] (each organic film spectrum is recorded from a surface slice $20 \mu \mathrm{m}$ wide) and allows us to record several spectra on not-irradiated surface regions just by shifting horizontally the sample. Diffraction patterns both in specular out-of-plane and in Grazing Incidence Diffraction (GID) geometries have been recorded by means of a NaI scintillator point detector.

\section{Results and Discussion}

\subsection{Pattern vs. Film Thickness: from Sub-Monolayer to Bulk}

The selective growth of $6 \mathrm{~T}$ molecules was investigated with respect to the nominal deposited thickness $t$ (expressed in $\AA$ and ML), as measured by the QCM. To study the nucleation stage, firstly we limit our investigations to the early stage of growth $(t=10, \AA \approx 0.35 \mathrm{ML})$, i.e., at low coverage $\Theta \leq 40 \%$, when neither coalescence nor Ostwald ripening of the islands are observed [32]. As shown in Figure 3,6T molecules were deposited on a tailored substrate with oxide stripes on the half of the $\mathrm{SiO}_{x}$ surface. In the P part (Figure 3b), they nucleate and grew selectively on the top surface of the stripes, resulting in stretched islands compared with the rounded ones grown on the NP part (Figure 3a). The 
selective growth arises from the interplay between kinetic growth parameters and preferential interactions with the patterned surface [12].

On $\mathrm{P}$ surface, the surface coverage $\Theta$ is reduced $\left(\Theta_{\mathrm{NP}} \approx 40 \%, \Theta_{\mathrm{P}} \approx 20 \%\right)$ as well as the number of islands $N$ (from $N_{\mathrm{NP}} \approx 50$ to $N_{\mathrm{P}} \approx 30$ ). In either case, the height of the islands is constant to (40 \pm 4$) \AA$, so an enhanced molecular diffusion induced by the pattern should be excluded. As reported elsewhere [33], this height value exceeds largely the length of the 6T molecule measured by means X-ray [30] (24 $\AA$ ), indicating the presence of a second molecular layer on the top of the 6T islands [34]. Accordingly, $\Theta$ and $N$ differences might be ascribed either to the geometry/morphology of the $\mathrm{SiO}_{x}$ pattern or the molecular growth mechanism.

In the matter of the geometrical shape of the $\mathrm{SiO}_{x}$ pattern, it is composed by an array of stripes with $\lambda \approx 1.4 \mu \mathrm{m}, \mathrm{FWHM} \approx 500 \mathrm{~nm}, h=(20 \pm 6) \AA$, top width $\approx 460 \mathrm{~nm}$ (top surface of the stripes) and basal width $\approx 650 \mathrm{~nm}$ (interstitial surface between stripes). The oblique angle between top and basal vertexes is $\approx 0.3^{\circ}$, which is too small for assigning the observed $\Theta$ difference to an image artefact [35].

Concerning the morphology of the $\mathrm{SiO}_{x}$ pattern, the roughness $R_{q}$ on both top and basal surfaces was $\approx 3 \AA$ and $\approx 12 \AA$, respectively [36]. As reported elsewhere [37], the molecular diffusion is favoured on low roughness surfaces (top surface of the stripes) with respect to high roughness surface (basal surface). On the basis of this simple morphological argument, the stretched shape of 6T islands can be explained, but neither geometrical nor morphological aspects of the $\mathrm{SiO}_{x}$ pattern are responsible for the $\Theta$ and $N$ decreasing.

On the other hand, the molecular growth mechanism depends on both surface chemical composition [19] and growth parameters ( $r$ [32] and $T_{\text {sub }}$ [21]). In our experiments, $r$ and $T_{\text {sub }}$ were kept constant (cf. 2.2). As shown by both Spectrophotometer and X-ray Photoemission Spectroscopy (XPS) measurements [16], the oxide fabricated by PLAO has an average thickness of $\approx 130 \AA$ (the pristine oxide has $\approx 20 \AA$ ), exhibits the $30 \%$ of porosity more than the thermal one and it is mainly composed by $\mathrm{Si}$ dioxide $\left(\mathrm{SiO}_{2}\right)$ with some small contributions to other Si chemical states. Its water contact angle (WCA) [38] is $\approx 100^{\circ}$ while the one measured on the native Si oxide is $\approx 60^{\circ}$ (for $\mathrm{Si}$ specification cf. 2.2). The increase of the WCA from native to anodized oxide reflects a surface chemical composition change. Accordingly, the islands density $N$ (and consequently $\Theta$ ) is reduced because of the lower sticking coefficient $[19,39]$.

For increasing film thickness, the selective effect of the pattern was investigated by X-ray scattering and AFM. X-ray scattering measurements in-situ and in real-time were performed on NP and P (stripes with $\lambda=1.4 \mu \mathrm{m}, \mathrm{FWHM} \approx 500 \mathrm{~nm}$ and $h=(22 \pm 2) \AA) \mathrm{Si} / \mathrm{SiO}_{x}$ substrates $\left(1 \mathrm{~cm}^{2}\right)$. The sample shutter was moved laterally ( 6 steps of about $35 \mathrm{~mm}$ ) to screen partially the P surface to the molecular beam (film thickness sequence: 13, 33, 45, 52, 81 and $108 \AA$ ), thus the film thickness increases gradually from $13 \AA$ ( $\approx 0.5 \mathrm{ML}$ - thinner area) to $108 \AA$ ( $\approx 4.5 \mathrm{ML}$-thickest area). The shutter of the Knudsen cell was closed when additional X-ray measurements were required.

Firstly, 6T films deposited on $\mathrm{NP} \mathrm{SiO}_{x}$ substrate have been investigated as a reference. Figure 4a

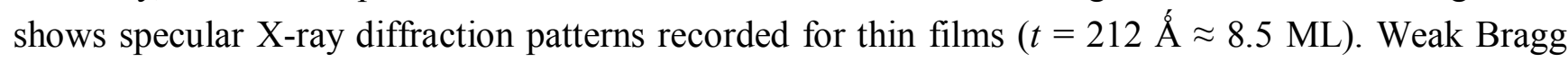
peaks were recorded for 3.5 ML thick film, which became quite pronounced for film $8 \mathrm{ML}$ thick. The presence of the only (h00) reflections and their angular positions showed a monoclinic unit cell for 6T

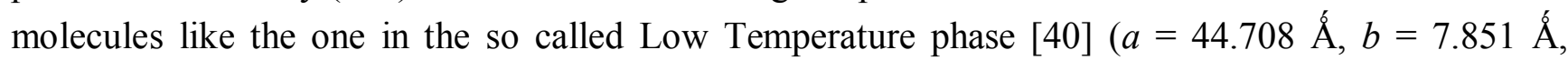
$c=6.029 \AA$, $\beta=90.76^{\circ}$ ). Along the direction orthogonal to the surface, molecules stack preferentially 
along their long axis, almost perpendicular to the substrate. The film consists of crystallites whose vertical domain size is of the same order of the film thickness (8.5 ML thick films), as estimated by the FWHM of the diffraction peak [41]. Additional information has been obtained analyzing the GID patterns performed with an incident angle $\alpha_{i}=0.1^{\circ}$, just above the critical angle of the silicon substrate (Figure 4b).

Figure 4. Series of XRD patterns collected in (a) specular and (b) GID geometries of 6T thin films deposited on $\mathrm{NP} \mathrm{Si} / \mathrm{SiO}_{x}$ with thickness ranging from 1 to $8.5 \mathrm{MLs}$. The GID were performed with $\alpha_{i}=0.1^{\circ}$.
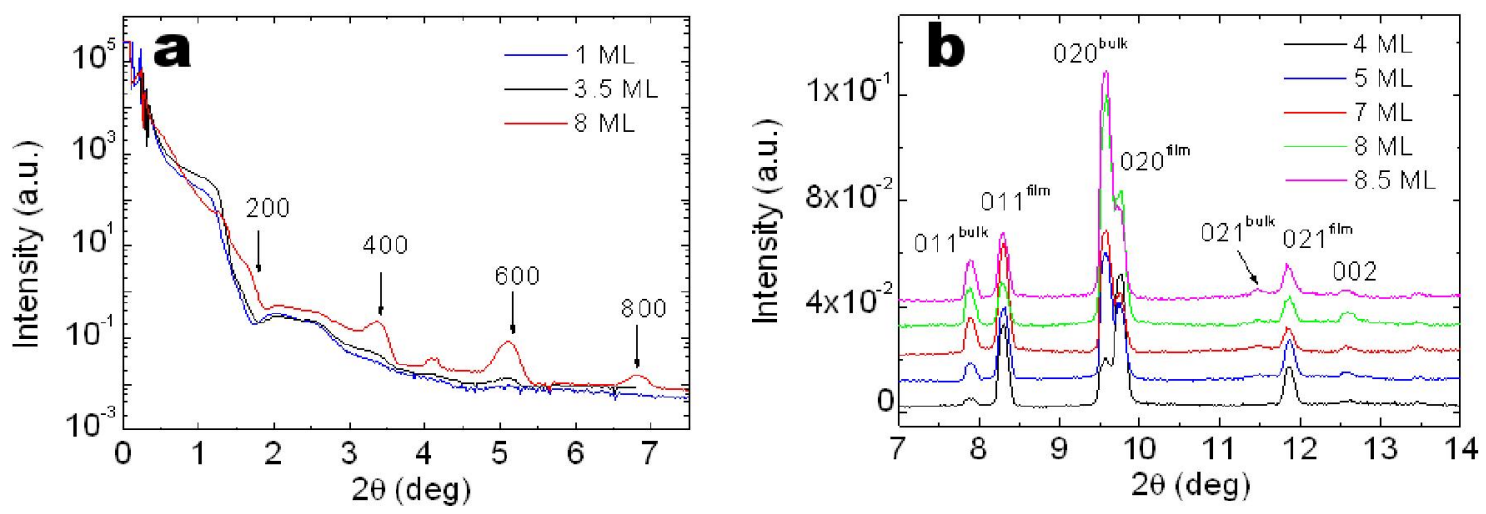

The presence of $(0 \mathrm{kl})$ reflections confirms the crystallite preferential orientation with the (bc) plane parallel to the substrate surface. The peaks arising from thin film and bulk phases coexist for all the film thicknesses. For thinner film, the film phase largely dominates while it progressively decreases to the bulk one for increasing thickness.

Combining out-of plane and in-plane geometries for X-ray scattering we have investigated the

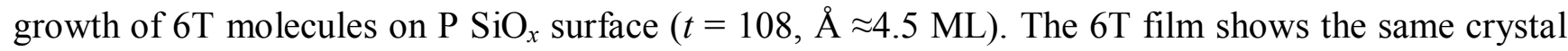
structure, preferential orientation and co-presence of film and bulk phases, as determined for the film deposited on $\mathrm{NP} \mathrm{SiO}_{x}$ substrate. In order to verify a possible orientation with respect to the $\mathrm{SiO}_{x}$ stripes, azimuthal scans have been performed in GID geometry, i.e., scattering intensity has been acquired by keeping the detector at the Bragg angle of the (011) and (020) reflections and turning the sample around the direction orthogonal to the surface [42]. The intensity invariance along these scans indicates no preferential orientation with respect to the stripes, differently from what observed on $6 \mathrm{~T}$ film grown on $\mathrm{SiO}_{2}$ grooves $(\lambda=400 \mathrm{~nm}$, width $200 \mathrm{~nm}$ and depth $100 \AA)$ where the packing of molecules shows graphoepitaxy [43] with the $b$ axis of the cell parallel to the groove direction.

Conversely to X-ray measurements, ex-situ AFM measurements on the $6 \mathrm{~T}$ film grew on the $\mathrm{P}$ surface show a selective growth of the film up to $\approx 3.2 \mathrm{ML}$, thickness for which the pattern effect disappears (Figure 5a). For film thicknesses ranging from $13 \AA \approx 0.5 \mathrm{ML}$ to $81 \AA \approx 3.2 \mathrm{ML}, 6 \mathrm{~T}$ islands were aligned along the stripes, moving away from the stripes direction of $\eta \approx 1^{\circ}$ (polar plot of Figure 5a,b). On the contrary, islands grown on a NP surface region showed a random spatial distribution (Figure $5 \mathrm{c}$ and its polar plot). The order parameter $\mathrm{S}$ is constant to $\approx 0.5$ for all thicknesses (Figure 5d) while it decreases about the 50\% $(\approx 0.25)$ for $6 \mathrm{~T}$ islands grown on the NP surface (Figure $5 \mathrm{~d}$-depicted bar not-labeled in the $\mathrm{X}$-axis). As described above, $6 \mathrm{~T}$ islands nucleate and grew preferentially on the top surface of the stripes (that cover $\approx 50 \%$ of the total surface area) and their 
coverage $\Theta_{\text {stripe }}$ increases in the early stage of the growth $\left(t=13 \AA \approx 0.5 \mathrm{ML} \rightarrow \Theta_{\text {stripe }}=50 \%\right.$, $33 \AA \approx 1.4 \mathrm{ML} \rightarrow \Theta_{\text {stripe }}=63 \%$ ) till the soil of $45 \AA \approx 1.9 \mathrm{ML}$ where $\Theta_{\text {stripe }}$ is $100 \%$.

Figure 5. AFM images of $6 \mathrm{~T}$ films $31 \AA$ thick grown on $\mathrm{P}$ surface (a) and $13 \AA$ thick grown on NP surface (c). The islands alignment along stripes was evaluated by means of both the angle $\eta(\mathbf{b})$ and the order parameter (d). Inset figure shows $\eta$ for each imaged 6T islands for P (a) and NP (c) surfaces.
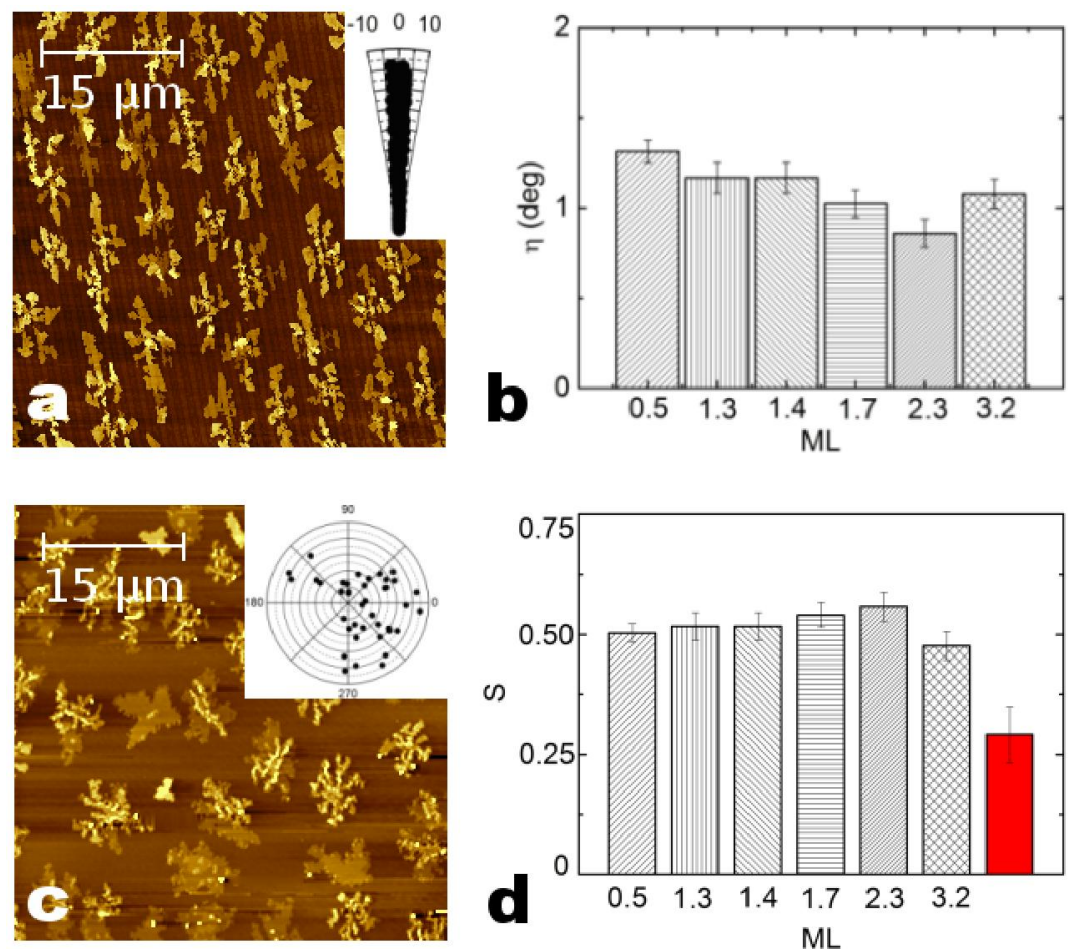

\subsection{Thermal Annealing and Thermal Stability of 6 T Films}

For every 6T film thicknesses, ex-situ AFM images show 6T crystals grew on the surface (Figure 5a). Their average lateral size increases (from 1 to $5 \mu \mathrm{m}^{2}$ ) with the film thickness (from $13 \AA \approx 0.5 \mathrm{ML}$ to $81 \AA \approx 3.2 \mathrm{ML}$ ). As reported in literature [44], the growth of $6 \mathrm{~T}$ crystals is due to the thermal annealing of the film during the growth process. In particular, the annealing process promotes a morphological transition of the film from grains to lamellae (highly crystalline aggregates). In our case, the time spent for X-ray measurements during the growth $(13 \mathrm{~h})$ is equivalent to an annealing process of the $6 \mathrm{~T}$ film at $120^{\circ} \mathrm{C}$. Moreover, the selective growth induced by the $\mathrm{SiO}_{x}$ pattern addresses further a lamellae growth [21].

$6 \mathrm{~T}$ films with lamellae morphology can be obtained by either reducing the deposition rate [45] or increasing the substrate temperature up to $150{ }^{\circ} \mathrm{C}$ [46]. Accordingly, the lamellae growth was investigated for a $6 \mathrm{~T}$ film deposited on identical $\mathrm{P} \mathrm{SiO}_{x}$ surface held to $120{ }^{\circ} \mathrm{C}$. The film thickness ( $61 \AA \approx 2.5 \mathrm{ML})$ was comparable to the previous ones but grown with lower both deposition rate $(r=0.007 \AA / \mathrm{s})$ and thermal annealing time $(3 \mathrm{~h})$. The annealing/pattern effect was successfully reproduced and $6 \mathrm{~T}$ crystals with lateral up to $20 \mu \mathrm{m}^{2}$ were grown on the patterned surface (Figure 6a). The crystallites are aligned along the stripes direction and show strong anisotropy in some preferential directions. As measured from AFM images, the polar plot shows 4 preferential angles $\left(30^{\circ}, 60^{\circ}, 90^{\circ}\right.$ 
and $120^{\circ}$ ) with respect to the stripes direction, accordingly to some preferential directions of growth ([010], [110], [100] and $[-1,1,0])[46]$. Those values are in agreement with previous result obtained on $6 \mathrm{~T}$ crystals grown on mica [46].

6T crystals were investigated by ex-situ X-ray measurements. Molecules are arranged in the bulk phase structure and they have very large lateral and vertical domain sizes, respectively $\mathrm{D}_{/ /}=800 \AA$ and $\mathrm{D} \perp=483 \AA$ (as estimated from the FWHM of the 011 and 200 peaks reported in Figure 6b,c).

Figure 6. (a) $6 \mathrm{~T}$ crystallites on $\mathrm{P} \mathrm{SiO}_{x}$ surfaces (arrows show the stripes direction). 3D topographic images of a $6 \mathrm{~T}$ film $52 \AA$ thick and deposited at $r \approx 0.04 \AA / \mathrm{s}$ (Left) and a $6 \mathrm{~T}$ film $61 \AA$ thick and deposited at $\approx 0.007 \AA / \mathrm{s}$ (Right). Specular (b) and GID (c) diffraction patterns recorded for $6 \mathrm{~T}$ deposited on $\mathrm{P} \mathrm{SiO}_{x}$ surface at $r \approx 0.007 \AA / \mathrm{s}$.
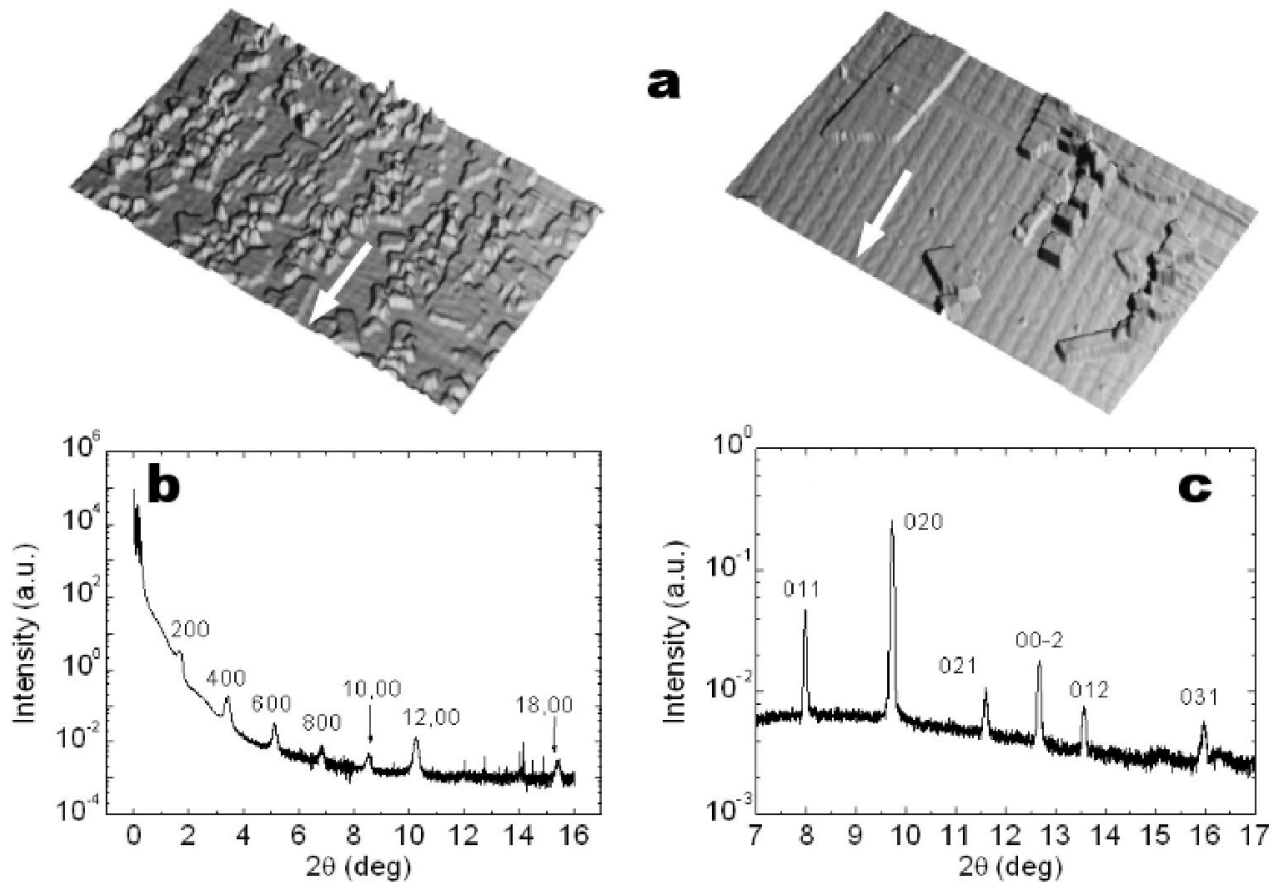

The thermal stability of the $6 \mathrm{~T}$ film after the growth process was investigated by AFM performed in air and for increasing $T_{\text {sub }}$ (RT, 50, 70, 80, 90, 110 and $\left.120^{\circ} \mathrm{C}\right)$. A sub-ML thick film (8 $\AA$ ) grown on $\mathrm{P} \mathrm{SiO}_{x}$ surface (stripes with $\lambda \approx 1.6 \mu \mathrm{m}, \mathrm{FWHM} \approx 580 \mathrm{~nm}, h=(13 \pm 2) \AA$ ) was chosen to stress as much as possible the morphological transformations of the film induced by the temperature.

As reported in literature, in air the $6 \mathrm{~T}$ polycrystalline powder melt at $305{ }^{\circ} \mathrm{C}[47,48]$, well above our operative conditions $\left(120{ }^{\circ} \mathrm{C}\right.$ at most). Moreover, in the temperature range $\mathrm{RT}-120$, polymeric thin films ( $<100 \mathrm{~nm}$ ) have shown de-wetting phenomena [49] induced by thermal fluctuations [50].

The thermal stability of $6 \mathrm{~T}$ film was firstly investigated by measuring the relative distances $d_{n}$ among 6T islands (where $\mathrm{n}$ is the ordinal number of islands). Each $d_{n}$ is the distance between the geometric centre of an island and the 6T crystal (Figure 7a). Regardless $T_{\text {sub }}, d_{n}$ are constant, viz. the geometrical positions of the islands on the $\mathrm{P}_{\mathrm{SiO}}$ surface are fixed.

In order to reach the thermal equilibrium (cf. 2.3), topographic images of $6 \mathrm{~T}$ film were recorded with the substrate kept to $T_{\text {sub }}$ for one hour. As a consequence, the film was subjected to a long post-annealing process in air ( 9 h: 6 temperatures- 6 h, plus 6 AFM images $-3 \mathrm{~h}$ ) which is, de facto, a 
thermal annealing. Usually, such post-processes improve the crystallographic order and change the morphological properties of the film [51]. The effect of this post-process can be evaluated by measuring the fractal dimension $D_{\mathrm{f}}$ that, in the case of sub-monolayer organic films [52], provides information on the growth process [53]. 6T islands have $D_{\mathrm{f}-\mathrm{NP}}=(1.310 \pm 0.006)$ and $D_{\mathrm{f}-\mathrm{P}}=(1.77 \pm 0.03)$ for NP and P surfaces, respectively and these values are constant with respect to $T_{\text {sub. Nevertheless, }}$ $D_{\mathrm{f}}$ suggests a morphological transition induced by either pattern and surface chemical composition (cf. 3.1): the growth mechanism evolves from a molecular diffusion at island edges [54] (formation of non-fractal structures) towards a diffusion limited aggregation scenario [55] (formation of fractal structures).

In spite of the invariance of $d_{n}$ and $D_{\mathrm{f}}$ to $T_{\text {sub }}$, the order parameter $\mathrm{S}$ changes abruptly between 90 and $100{ }^{\circ} \mathrm{C}$. As shown in Figure $7 \mathrm{~b}, \mathrm{~S}$ is $\approx 0.46$ up to $90{ }^{\circ} \mathrm{C}$ (in agreement with the previous value observed for increasing thickness) then it changes to $\approx 0.56$ in the temperature range $90-120{ }^{\circ} \mathrm{C}$. In addition, AFM images show a roughness increasing of the islands top surface from $\approx 2.8 \AA$ to $\approx 5.2 \AA$ (Figure 7c) [36]. The latter observation suggests the spinodal dewetting (SD) of the 6T film. In $\mathrm{SD}$, height variation patterns were created due to the film undulations induced by $T_{\text {sub }}$ [56]. In our case, SD was activated above $90{ }^{\circ} \mathrm{C}$ and $6 \mathrm{~T}$ molecules were reorganized towards the stripes (arrows in Figure 7c). As a consequence, on one hand, the top surface roughness of $6 \mathrm{~T}$ islands increases while, on the other hand, the molecules moved from the basal surface caused S increases.

Figure 7. (a) Topographic image of $6 \mathrm{~T}$ film recorded at RT. Islands relative distances $d_{n}$ (with $n=1, \ldots 5$ ) are sketched. (b) Order parameter vs. $T_{\text {sub }}$ (c) 3D topographic images of an island recorded at RT (left) and $120{ }^{\circ} \mathrm{C}$ (right). The top surface of $6 \mathrm{~T}$ island at $120{ }^{\circ} \mathrm{C}$ shows a clear roughening induced by SD.
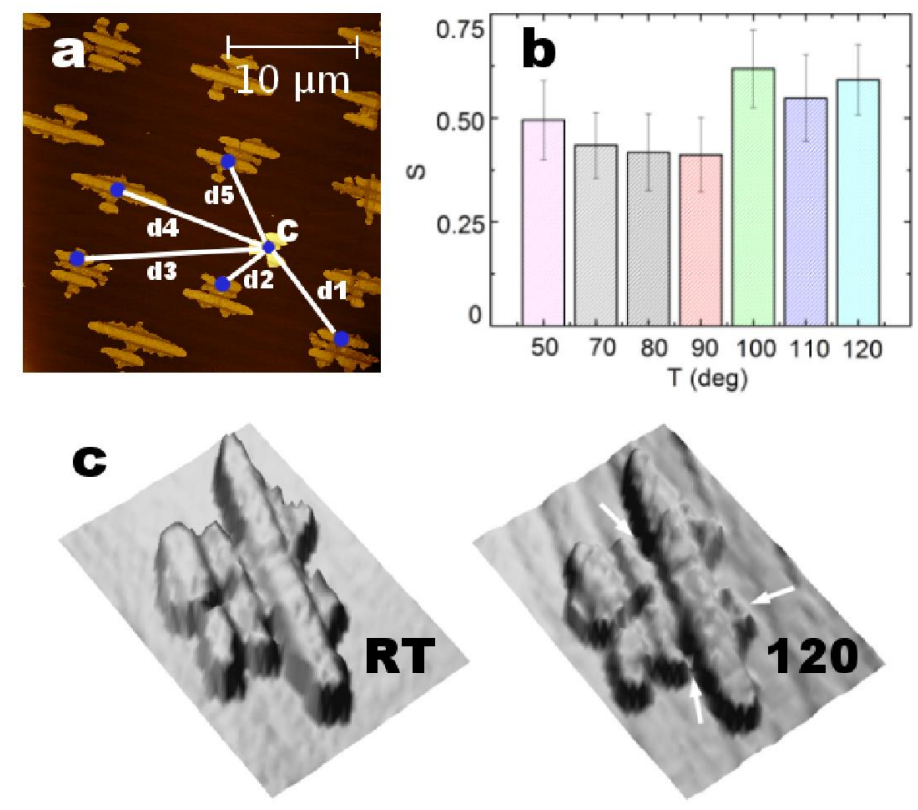

\subsection{Enhancement of the 6T Selective Growth by Means of Electric Field and Grid Pattern}

Electrostatic interactions between a charged substrate surface and organic molecules are used for controlled placement of nanoscale building blocks [57]. This was done by creating charge patterns, i.e., 
electrostatic templates, on the substrate surface and letting the organic molecules interact with the charge patterns.

In $\mathrm{SiO}_{x}$ patterns fabricated by local anodic oxidation, the existence of space charges has been proved [58], whereupon both topographical and electrical effects are responsible for the 6T arrangement on the pattern. In particular, the space charge influence is two-fold: on one hand, it enhances the placement of $6 \mathrm{~T}$ molecules while on the other hand it competes with the molecular diffusion driven by the substrate temperature. This is true especially where the space charge shows some non-homogeneity [59].

The latter problem should be solved to increase the amount of $6 \mathrm{~T}$ molecules placed on the pattern. For this reason, $\mathrm{P} \mathrm{SiO}_{x}$ substrates were investigated by Phase-Electrostatic Force Microscopy (Phase-EFM) [60]. The tip was grounded while the P substrate was biased. A series of EFM images (not shown here) are recorded for increasing bias, from 0 to $20 \mathrm{~V}$. The electrostatic contrast on top of the $\mathrm{SiO}_{x}$ pattern shows an increased charge homogeneity, which saturates at $15 \mathrm{~V}$.

In order to investigate the enhanced effect driven by the electrical potential, $6 \mathrm{~T}$ film $12 \AA$ thick $(\Theta \approx 50 \%)$ was grown on two identical $\mathrm{P} \mathrm{SiO}_{x}$ surfaces (stripes with $\lambda \approx 1.4 \mu \mathrm{m}, \mathrm{FWHM} \approx 550 \mathrm{~nm}$, $h=(22 \pm 1) \AA)$, one grounded and the other one $15 \mathrm{~V}$ biased. The surface coverage $\Theta$ was chosen just above the low coverage limit $(\Theta=40 \%)$ for reaching the islands coalescence through the electric field. As shown in Figure 8a,b, the surface coverage is enhanced because molecules are mostly placed on top of the stripes and they are covered $\left(\Theta_{\text {stripe }}\right)$ for about the $90 \%$ of their surface (Table 1$)$.

Figure 8. Topographic images of $6 \mathrm{~T}$ film on $\mathrm{P} \mathrm{SiO}_{x}$ surface (a) grounded and (b) biased; (c) complete molecular arrangement on $\mathrm{SiO}_{x}$ grid.
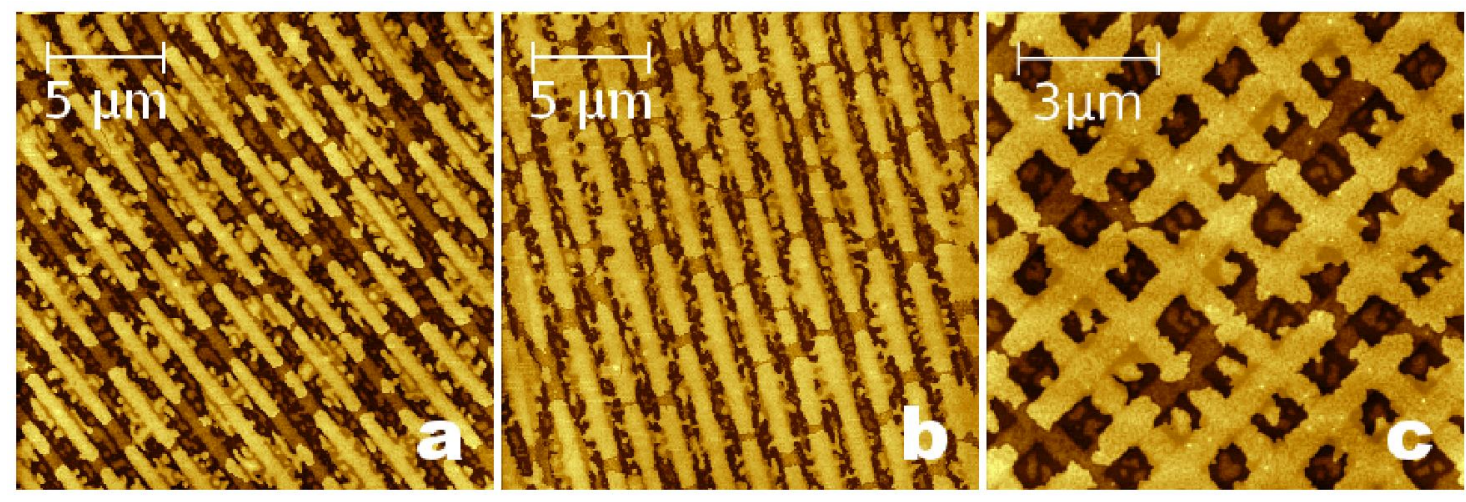

Table 1. Angle $\eta$ between $D_{\max }$ (cf. 2.4) and the stripes direction, order parameter $S$ and stripes surface coverage $\Theta_{P}$ for grounded and biased samples.

\begin{tabular}{cccc}
\hline & $\boldsymbol{\eta}\left(^{\circ}\right)$ & $\boldsymbol{S}$ & $\boldsymbol{\Theta}_{\text {stripe }}(\mathbf{\%})$ \\
\hline Ground & $0.81 \pm 0.08$ & $0.62 \pm 0.03$ & $73 \pm 5$ \\
$15 \mathrm{~V}$ & $0.6 \pm 0.1$ & $0.75 \pm 0.03$ & $86 \pm 4$ \\
\hline
\end{tabular}

As observed in Figure 8a, 6T film (12 A thick) grown on grounded surface shows stretched islands in which the part of the island residing on top of the stripes ranges from 1 to $5 \mu \mathrm{m}$. This range of lengths suggests another way to drive topographically the molecular arrangement using a pattern with comparable (or smaller) features. An almost complete arrangement of $6 \mathrm{~T}$ molecules was reached by using a grid with holes size $\approx 650 \mathrm{~nm}$ and bar width $\approx 500 \mathrm{~nm}$ (Figure $8 \mathrm{c}$ ). 


\section{Conclusions}

We have demonstrated a process for the fabrication of ordered low-dimensional structures of $6 \mathrm{~T}$ molecules on large area. The process is based on the integration of $\mathrm{SiO}_{x}$ substrates patterns fabricated by using the parallel local anodic oxidation technique, and the selective growth of molecular thin films. The main result is the control of $6 \mathrm{~T}$ islands anisotropy by topographic patterns; in such a way that we have successfully self-assembled both stripes and networks of 6T islands. Such morphological control is thickness-dependent and, on $\mathrm{SiO}_{x}$ stripes, it reaches $100 \%$ of covered stripes for $\approx 1.9$ ML thick film. For thicker film, the pattern effect disappears. The $\mathrm{SiO}_{x}$ pattern does not induce any $6 \mathrm{~T}$ crystalline deformation or changes in molecular orientation. Indeed, the crystalline structure presents the same features observed on 6T thin film grown on non-patterned substrate. Patterned 6T films are thermally stable and the best patterns coverage with a lower amount of deposited materials has been reached by enhancing the pattern effect with the electric field and different geometries (grid). Because molecules stand with their long axes almost orthogonal to the substrate, the present process is suitable to fabricate substrate with precise topographical and chemical patterns. Moreover, parallel arrays (or networks) of nanometer-size 6T stripes placed between two planar electrodes are suitable for systematic studies of charge transport in conjugated organic molecular wires.

\section{Acknowledgments}

The authors wish to thank Mauro Murgia and Paolo Mei for helping in the construction of the OMBD system and the PLAO machine, respectively. The work was supported by both NAIMO (NMP4, 500355) and ONE-P (FP7, 212311).

\section{References and Notes}

1. Koh, S.J. Strategies for controlled placement of nanoscale building blocks. Nanoscale Res. Lett. 2007, 2, 519-545.

2. Song, H.; Reed, M.A.; Lee, T. Single-molecule devices: Single molecule electronic devices. $A d v$. Mater. 2011, 23, 1583-1608.

3. Khajetoorians, A.A.; Wiebe, J.; Chilian, B.; Wiesendanger, R. Realizing all-spin-based logic operations atom by atom. Science 2011, 332, 1062-1064.

4. Shehu, A.; Quiroga, S.D.; D’Angelo, P.; Albonetti, C.; Borgatti, F.; Murgia, M.; Scorzoni, A.; Stoliar, P.; Biscarini, F. Layered distribution of charge carriers in organic thin film transistors. Phys. Rev. Lett. 2010, 104, 246602:1-246602:4.

5. Cavallini, M.; Albonetti, C.; Biscarini, F. Nanopatterning soluble multifunctional materials by unconventional wet lithography. Adv. Mater. 2009, 21, 1043-1053.

6. Melucci, M.; Zambianchi, M.; Favaretto, L.; Palermo, V.; Treossi, E.; Montalti, M.; Bonacchi, S.; Cavallini, M. Multicolor, large-area fluorescence sensing through oligothiophene-self-assembled monolayers. Chem. Commun. 2011, 47, 1689-1691. 
7. Melucci, M.; Favaretto, L.; Zanelli, A.; Cavallini, M.; Bongini, A.; Maccagnani, P.; Ostoja, P.; Derue, G.; Lazzaroni, R.; Barbarello, G. Thiophene-benzothiadiazole co-oligomers: Synthesis, optoelectronic properties, electrical characterization, and thin-film patterning. Adv. Funct. Mater. 2010, 20, 445-452.

8. Sirringhaus, H.; Shimoda, T. Inkjet printing of functional materials. MRS Bull. 2003, 28, 802-803.

9. Dinelli, F.; Murgia, M.; Levy, P.; Cavallini, M.; Biscarini, F.; de Leeuw, D.M. Spatially correlated charge transport in organic thin film transistors. Phys. Rev. Lett. 2004, 92, 116802:1-116802:4.

10. Dediu, V.A.; Hueso, L.E.; Bergenti, I.; Taliani, C. Spin routes in organic semiconductors. Nat. Mater. 2009, 8, 707-716.

11. Capelli, R.; Toffanin, S.; Generali, G.; Usta, H.; Facchetti, A.; Muccini, M. Organic light-emitting transistors with an efficiency that outperforms the equivalent light-emitting diodes. Nat. Mater. 2010, 9, 496-503.

12. Garcia, R.; Tello, M.; Moulin, J.F.; Biscarini, F. Size and shape controlled growth of molecular nanostructures on silicon oxide templates. Nano Lett. 2004, 4, 1115-1119.

13. Losilla, N.S.; Martinez, J.; Bystrenova, E.; Greco, P.; Biscarini, F.; García, R. Patterning pentacene surfaces by local oxidation nanolithography. Ultramicroscopy 2010, 110, 729-732.

14. Steudel, S.; Janssen, D.; Verlaak, S.; Genoe, J.; Heremans, P. Patterned growth of pentacene. Appl. Phys. Lett. 2004, 85, 5550-5552.

15. Brinkmann, M.; Graff, S.; Biscarini, F. Mechanism of nonrandom pattern formation of polar-conjugated molecules in a partial wetting regime. Phys. Rev. B 2002, 66, 165430:1-165430:8.

16. Albonetti, C.; Martinez, J.; Losilla, N.S.; Greco, P.; Cavallini, M.; Borgatti, F.; Montecchi, M.; Pasquali, L.; Garcia, R.; Biscarini, F. Parallel-local anodic oxidation of silicon surfaces by soft stamps. Nanotechnology 2008, 19, 435303, doi:10.1088/0957-4484/19/43/435303.

17. Cavallini, M.; Mei, P.; Biscarini, F.; García, R. Parallel writing by local oxidation nanolithography with submicrometer resolution. Appl. Phys. Lett. 2003, 83, 5286-5288.

18. Zhao, X.-M.; Xia, Y.N.; Whitesides, G.M. Soft lithographic methods for nano-fabrication. J. Mater. Chem. 1997, 7, 1069-1074.

19. Dinelli, F.; Moulin, J.-F.; Loi, M.A.; da Como, E.; Massi, M.; Murgia, M.; Muccini, M.; Biscarini, F.; Wie, J.; Kingshott, P. Effects of surface chemical composition on the early growth stages of alpha-sexithienyl films on silicon oxide substrates. J. Phys. Chem. B 2006, 110, 258-263.

20. We define a monolayer (ML) as a layer of ordered and closed packed molecules orthogonal to the surface that completely covers it $(\Theta=100 \%)$. A nominal ML is equivalent to $24 \AA$ of $6 \mathrm{~T}$ molecules deposited on the $\mathrm{SiO}_{x}$ substrate.

21. Viville, B.P.; Lazzaroni, R.; Bredas, J.L.; Moretti, P.; Samorí, P.; Biscarini, F. The influence of thermal annealing on the morphology of sexithienyl thin films. Adv. Mater. 1998, 57-60.

22. García, R.; Perez, R. Dynamic atomic force microscopy methods. Surf. Sci. Rep. 2002, 47, 197-301.

23. Alexeev, A. Study of monomolecular layers of azobenzene derivative by the scanning probe microscopy. Mater. Sci. Eng. C 2002, 22, 453-458.

24. Wu, Y.; Toccoli, T.; Koch, N.; Iacob, E.; Pallaoro, A.; Rudolf, P.; Iannotta, S. Controlling the early stages of pentacene growth by supersonic molecular beam deposition. Phys. Rev. Lett. 2007, 98, 076601:1-076601:4. 
25. Dinelli, F.; Albonetti, C.; Kolosov, O.V. Ultrasonic force microscopy: Detection and imaging of ultra-thin molecular domains. Ultramicroscopy 2011, 111, 267-272.

26. Necas, D.; Klapetek, P. Gwyddion Free Software. Available online: http:/gwyddion.net/ (accessed on 31 August 2011).

27. Klapetek, P.; Necas, D.; Anderson, C. Gwyddion User Guide, version 2009-11-11. Available online: http:// gwyddion.net (accessed on 5 September 2011).

28. Straley, J.P. Ordered phases of a liquid of biaxial particles. Phys. Rev. 1974, 10, 1881-1887.

29. Rasband, W.S. Image J. Available online: http://rsbweb.nih.gov/ij/ (accessed on 31 August 2011).

30. Moulin, J.-F.; Dinelli, F.; Massi, M.; Albonetti, C.; Kshirsagar, R.; Biscarini, F. In situ X-ray synchrotron study of organic semiconductor ultra-thin films growth. Nucl. Instrum. Methods Phys. Res. Sect. B 2006, 246, 122-126.

31. Chua, L.-L.; Dipankar, M.; Sivaramakrishnan, S.; Gao, X.; Qi, D.; Wee, A.T.S.; Ho, P.K.H. Large damage threshold and small electron escape depth in X-ray absorption spectroscopy of a conjugated polymer thin film. Langmuir 2006, 22, 8587-8594.

32. Pratontep, S.; Brinkmann, M.; Nüesch, F.; Zuppiroli, L. Correlated growth in ultrathin pentacene films on silicon oxide: Effect of deposition rate. Phys. Rev. B 2004, 69, 165201, doi:10.1103/ PhysRevB.69.165201.

33. Martinez, N.F.; Patil, S.; Lozano, J.R.; Garcia, R. Enhanced compositional sensitivity in atomic force microscopy by the excitation of the first two flexural modes. Appl. Phys. Lett. 2006, 89, 153115, doi:10.1063/1.2360894.

34. Martínez, N.F.; Kamiński, W.; Gómez, C.J.; Albonetti, C.; Biscarini, F.; Pérez, R.; García, R. Molecular scale energy dissipation in oligothiophene monolayers measured by dynamic force microscopy. Nanotechnology 2009, 20, 434021, doi:10.1088/0957-4484/20/43/434021.

35. The surface coverage $\Theta$ is the sum of the islands areas projected on the $\mathrm{SiO}_{x}$ plane therefore an oblique angle decrease the projected area (cf. 2.4).

36. Standardized one-dimensional $R_{\mathrm{q}}$ is evaluated from line profiles (10 $\mu \mathrm{m}$ long and 15 lines thick) taken along both top and basal surfaces.

37. Besana, D.; Borghesi, A.; Campione, M.; Sassella, A.; Tubino, R.; Moret, M.; Rinaldi, R.; Garnier, F. Influence of the substrate on the growth of [alpha],[omega]-dihexyl-quaterthiophene thin films by organic molecular beam deposition. J. Cryst. Growth 2002, 235, 241-247.

38. Matsumoto, Y. Novel prevention method of stiction using silicon anodization for SOI structure. Sens. Actuators A 1999, 72, 153-159.

39. Forrest, S.R. Ultrathin organic films grown by organic molecular beam deposition and related techniques. Chem. Rev. 1997, 97, 1793-1896.

40. Horowitz, G.; Bachet, B.; Yassar, A.; Lang, P.; Demanze, F.; Fave, J.L.; Garnier, F. Growth and characterization of sexithiophene single-crystals. Chem. Mater. 1995, 7, 1337-1341.

41. Sherrer, P. Nachrichten von der Gesellschaft der Wissenschaften zu Göttingen; Göttinger Digitalisierungszentrum: Goettingen, Germany, 1918; pp. 98-100.

42. Milita, S.; Dionigi, C.; Borgatti, F.; Lazar, A.N.; Porzio, W.; Destri, S.; Wermeille, D.; Felici, R.; Andreasen, J.W.; Nielsen, M.M.; et al. Crystallization of organic semiconductor molecules in nanosized cavities: Mechanism of polymorphs formation studied by in situ XRD. J. Phys. Chem. C 2008, 112, 12177-12183. 
43. Ikeda, S.; Saiki, K.; Wada, Y.; Inaba, K.; Ito, Y.; Kikuchi, H.; Terashima, K.; Shimada, T. Graphoepitaxy of sexithiophene and orientation control by surface treatment. J. Appl. Phys. 2008, 103, 084313:1-084313:9.

44. Biscarini, F.; Samorí, P.; Greco, O.; Zamboni, R. Scaling behavior of anisotropic organic thin films grown in high vacuum. Phys. Rev. Lett. 1997, 78, 2389-2392.

45. Muccini, M.; Murgia, M.; Biscarini, F. Morphology controlled energy transfer in conjugated molecular thin films. Adv. Mater. 2001, 13, 355-358.

46. Biscarini, F.; Zamboni, R.; Samorì, P.; Ostoja, P.; Taliani, C. Growth of conjugated oligomer thin films studied by atomic-force microscopy. Phys. Rev. B 1995, 52, 14868-14877.

47. Taliani, C.; Zamboni, R.; Ruani, G.; Rossini, S.; Lazzaroni, R. New rigid rod liquid-crystal molecule precursor of conjugated polymers: Alpha-sexithienyl. J. Mol. Electron. 1990, 6, 225-226;

48. Pizzirusso, A.; Savini, M.; Muccioli, L.; Zannoni, C. An atomistic simulation of the liquid-crystalline phases of sexithiophene. J. Mater. Chem. 2011, 21, 125-133.

49. Moulin, J.-F.; Kengne, J.C.; Kshirsagar, R.; Cavallini, M.; Biscarini, F.; León, S.; Zerbetto, F.; Bottari, G.; Leigh, D.A. Self-organization of rotaxane thin films into spatially correlated nanostructures: Morphological and structural aspects. J. Am. Chem. Soc. 2006, 128, 526-532.

50. Reiter, G. Unstable thin polymer films: Rupture and dewetting processes. Langmuir 1993, 9, 1344-1351.

51. De Luca, G.; Pisula, W.; Credgington, D.; Treossi, E.; Fenwick, O.; Lazzerini, G.M.; Dabirian, R.; Orgiu, E.; Liscio, A.; Palermo, V.; et al. Non-conventional processing and post-processing methods for the nanostructuring of conjugated materials for organic electronics. Adv. Funct. Mater. 2011, 21, 1279-1295.

52. The fractal dimension $D_{\mathrm{f}}$ is the scaling exponent of the power law between the perimeter $P$ and the area $A$ of $6 \mathrm{~T}$ islands $P \propto A^{\frac{D_{f}-1}{2}}$.

53. Meyer zu Heringdorf, F.J.; Reuter, M.C.; Tromp, R.M. Growth dynamics of pentacene thin films. Nature 2001, 412, 517-520.

54. Amar, J. Kinetics of submonolayer epitaxial growth. Comput. Phys. Commun. 2002, 146, 1-8.

55. Witten, T.A.; Sander, L.M. Diffusion-limited aggregation, a kinetic critical phenomenon. Phys. Rev. Lett. 1981, 47, 1400-1403.

56. Xie, R.; Karim, A.; Douglas, J.; Han, C.; Weiss, R. Spinodal dewetting of thin polymer films. Phys. Rev. Lett. 1998, 81, 1251-1254.

57. Losilla, N.S.; Martínez, J.; García, R. Large area nanoscale patterning of silicon surfaces by parallel local oxidation. Nanotechnology 2009, 20, 475304, doi:10.1088/0957-4484/20/47/475304.

58. Chiesa, M.; Garcia, R. Nanoscale space charge generation in local oxidation nanolithography. Appl. Phys. Lett. 2010, 96, 263112.

59. Losilla, N.S.; Oxtoby, N.S.; Martinez, J.; Garcia, F.; Garcia, R.; Mas-Torrent, M.; Veciana, J.; Rovira, C. Sub-50 nm positioning of organic compounds onto silicon oxide patterns fabricated by local oxidation nanolithography. Nanotechnology 2008, 19, 455308, doi:10.1088/09574484/19/45/455308. 
60. Annibale, P.; Albonetti, C.; Stoliar, P.; Biscarini, F. High-resolution mapping of the electrostatic potential in organic thin-film transistors by phase electrostatic force microscopy. J. Phys. Chem. A 2007, 111, 12854-12858.

(C) 2011 by the authors; licensee MDPI, Basel, Switzerland. This article is an open access article distributed under the terms and conditions of the Creative Commons Attribution license (http://creativecommons.org/licenses/by/3.0/). 